\title{
Analysis of Tangential Slot Blowing on F/A-18 Isolated Forebody
}

\author{
Ken Gee, ${ }^{*}$ Yehia M. Rizk, $\dagger$ and Lewis B. Schiff $\ddagger$ \\ NASA Ames Research Center, Moffett Field, California 94035-1000
}

\begin{abstract}
The generation of significant side forces and yawing moments on an F/A-18 fuselage through tangential slot blowing is analyzed using computational nuid dynamics. The effects of freestream Mach number, jet exit conditions, jet length, and jet location are studied. The effects of over- and underblowing on force and moment production are analyzed. Non-time-accurate solutions are obtained to determine the steady-state side forces, yawing moments, and surface pressure distributions generated by tangential slot blowing. Time-accurate solutions are obtained to study the force onset time Iag of tangential slot blowing. Comparison with available experimental data from full-scale wind-tunnel and subscale wind-tunnel tests are made. This computational analysis complements the experimental results and provides a detailed understanding of the effects of tangential slot blowing on the flowfield about the isolated F/A-18 forebody. Additionally, it extends the slot-blowing database to transonic maneuvering Mach numbers.
\end{abstract}

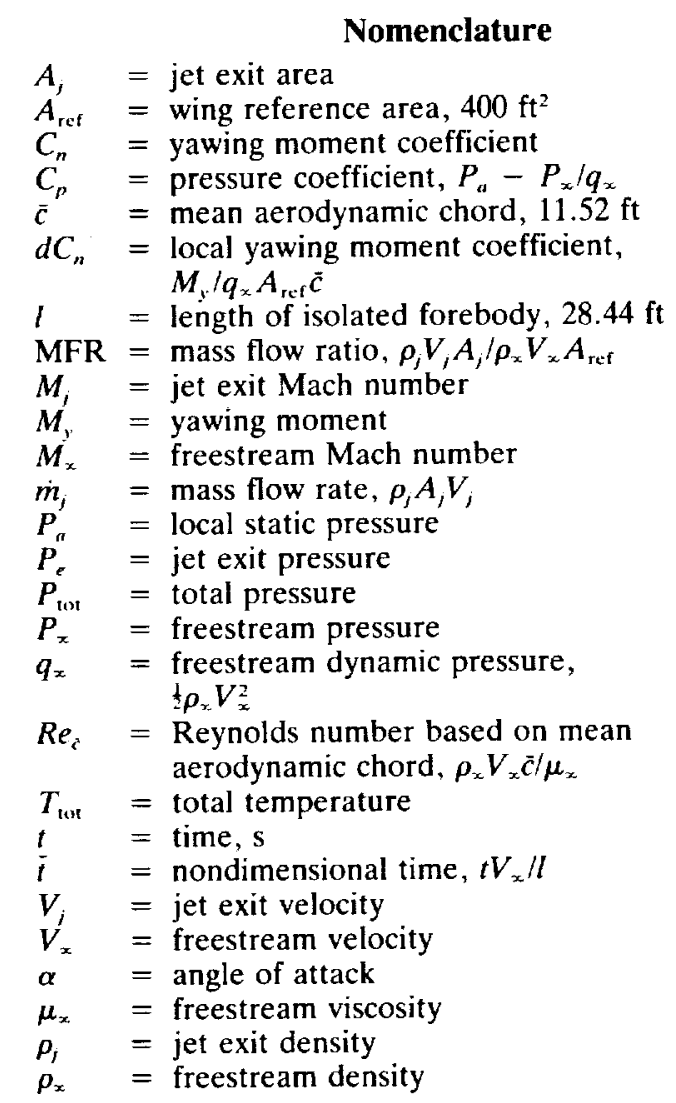

Presented as Paper 94-1831 at the AIAA 12th Applied Aerodynamics Conference, Colorado Springs, CO, June 20-23, 1994; received Aug. 10, 1994; revision received March 12, 1995; accepted for publication March 15, 1995. Copyright (C) 1995 by the American Institute of Aeronautics and Astronautics, Inc. No copyright is asserted in the United States under Title 17, U.S. Code. The U.S. Government has a royalty-free license to exercise all rights under the copyright claimed herein for Governmental purposes. All other rights are reserved by the copyright owner.

*Research Scientist, MCAT Institute, M/S 258-1. Member AIAA.

†Research Scientist. Associate Fellow AIAA.

$\ddagger$ Special Assistant for High Alpha Technology. Associate Fellow AIAA.
Introduction

$7 \mathrm{HE}$ use of pneumatic forebody flow control on aircraft flying at high angle of attack has been a topic of aerodynamic research over the past several years. The flowfield about an aircraft at high incidence is characterized by crossflow separations of the boundary layer, which then roll up to form vortices. At high angle of attack these vortices may become asymmetric, creating a side force and yawing moment on the aircraft, which can cause an uncontrolled departure of the aircraft from its intended flight path. Furthermore, flight at high angle of attack immerses the vertical tails in the wake of the fuselage and wing, reducing the effectiveness of these control surfaces. In order to provide the necessary control power to the pilot to maintain controlled flight, new methods of generating control forces and moments must be developed.

One such method under investigation is forebody tangential slot blowing. ${ }^{1.2}$ In this method, a thin slot is located near the tip of the nose of an aircraft from which air is ejected tangential to the nose surface (Fig. 1). The jet remains attached to the surface due to the Coanda effect and eventually separates. The jet alters the flowfield about the aircraft, which in turn generates a side force and yawing moment. This side force and yawing moment may then be used by the pilot to control the aircraft at high angle of attack.

Both experimental and computational investigations have been used to analyze the effectiveness of tangential slot blowing on the F/A-18. Experiments have been conducted on subscale models in water tunnels ${ }^{3}$ and wind tunnels, ${ }^{4}$ and on a full-scale model in a wind tunnel..$^{5}$ Computational investigations have been conducted on both the isolated F/A-18 forebody ${ }^{2.6}$ and on the full aircraft geometry. "These investigations have shown tangential slot blowing to be a viable

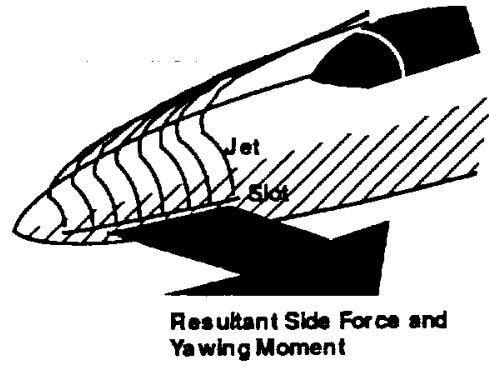

Fig. 1 Schematic of forebody tangential slot blowing concept. 
NASA/TM-95- 207300

$1 N-02-T M$

Analysis of Tangential Slot Blowing
on F/A-18 Isolated Forebody
Ken Gee, Yehia M. Rizk, Lewis B. Schiff

Reprinted from

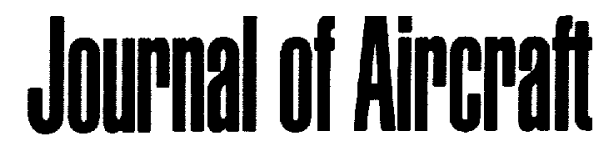

Volume 32, Number 5, Pages 1040-1046

DALAA.

A publication of the

American Institute of Aeronautics and Astronautics, Inc.

370 L'Enfant Promenade, SW

Washington, DC 20024-2518 
Table I Jet exit conditions used in computational study

\begin{tabular}{|c|c|c|c|c|c|c|}
\hline$M$. & $\begin{array}{l}\dot{m}_{j} \\
\mathrm{Ib} / \mathrm{s}\end{array}$ & $\begin{array}{l}\text { MFR, } \\
\times 10^{\cdots 3} \\
\end{array}$ & $M_{j}$ & $\begin{array}{c}P_{\text {tor, }} \\
\text { lb/in. }\end{array}$ & $\begin{array}{c}T_{\mathrm{tol}}, \\
{ }^{\circ} \mathrm{R}\end{array}$ & $P_{\mathrm{e}} / P_{\mathrm{at}}$ \\
\hline \multicolumn{7}{|c|}{ 16-in. slot starting 11 in. from the nose $(16-11$ in. slot) } \\
\hline 0.243 & 0.056 & 0.015 & 0.125 & 5.65 & 402 & 1.00 \\
\hline 0.243 & 0.111 & 0.03 & 0.248 & 5.83 & 405 & 1.00 \\
\hline 0.243 & 0.224 & 0.06 & 0.50 & 6.63 & 420 & 1.00 \\
\hline 0.243 & 0.432 & 0.12 & 0.96 & 10.14 & 473 & 1.00 \\
\hline 0.243 & 0.668 & 0.18 & 1.00 & 15.76 & 480 & 1.49 \\
\hline 0.243 & 0.868 & 0.24 & 1.00 & 20.49 & 480 & 1.94 \\
\hline 0.400 & 0.187 & 0.03 & 0.43 & 6.15 & 415 & 1.00 \\
\hline 0.400 & 0.368 & 0.06 & 0.85 & 8.68 & 460 & 1.00 \\
\hline 0.400 & 0.714 & 0.12 & 1.00 & 16.84 & 480 & 1.64 \\
\hline 0.400 & 1.098 & 0.18 & 1.00 & 25.90 & 480 & 2.53 \\
\hline 0.400 & 1.427 & 0.24 & 1.00 & 33.68 & 480 & 3.29 \\
\hline 0.700 & 0.323 & 0.03 & 0.76 & 7.78 & 447 & 1.00 \\
\hline 0.700 & 0.639 & 0.06 & 1.00 & 15.10 & 480 & 1.50 \\
\hline 0.700 & 1.248 & 0.12 & 1.00 & 29.44 & 480 & 2.93 \\
\hline 0.700 & 1.871 & 0.18 & 1.00 & 44.17 & 480 & 4.40 \\
\hline 0.700 & 2.495 & 0.24 & 1.00 & 58.89 & 480 & 5.86 \\
\hline
\end{tabular}

24-in. slot starting 3 in. from the nose (24-3 in. slot)

\begin{tabular}{llllrrr}
0.243 & 0.056 & 0.015 & 0.081 & 5.62 & 401 & 1.00 \\
0.243 & 0.111 & 0.03 & 0.162 & 5.69 & 403 & 1.00 \\
0.243 & 0.224 & 0.06 & 0.325 & 6.04 & 409 & 1.00 \\
0.243 & 0.432 & 0.12 & 0.63 & 7.36 & 432 & 1.00 \\
0.243 & 0.668 & 0.18 & 0.96 & 10.21 & 475 & 1.00 \\
0.243 & 0.868 & 0.24 & 1.00 & 13.33 & 480 & 1.26 \\
0.400 & 0.187 & 0.03 & 0.28 & 5.71 & 407 & 1.00 \\
0.400 & 0.368 & 0.06 & 0.55 & 6.67 & 425 & 1.00 \\
0.400 & 0.714 & 0.12 & 1.00 & 10.97 & 480 & 1.07 \\
0.400 & 1.098 & 0.18 & 1.00 & 16.94 & 480 & 1.66 \\
0.400 & 1.427 & 0.24 & 1.00 & 21.94 & 480 & 2.14 \\
0.700 & 0.323 & 0.03 & 0.50 & 6.25 & 420 & 1.00 \\
0.700 & 0.639 & 0.06 & 1.00 & 9.86 & 480 & 1.01 \\
0.700 & 1.248 & 0.12 & 1.00 & 19.10 & 480 & 1.92 \\
0.700 & 1.871 & 0.18 & 1.00 & 28.75 & 480 & 2.88 \\
0.700 & 2.495 & 0.24 & 1.00 & 38.40 & 480 & 3.85 \\
\hline \hline
\end{tabular}

method of generating side force and yawing moment on an aircraft flying at high angle of attack at relatively low freestream Mach numbers. To date, only low freestream Mach numbers have been investigated experimentally, due to the limitations of the facilities used. Similarly, previous computational studies have only been carried out at low freestream Mach numbers to compare with the experimental data.

However, a maneuvering fighter may attain high-angleof-attack flight at higher Mach numbers. The capability of forebody tangential slot blowing at higher freestream Mach numbers is not well understood. To develop such an understanding, a computational investigation is presented that analyzes the efficiency of tangential slot blowing at higher freestream Mach numbers. The numerical method employed has been shown to produce good results at the lower Mach numbers when compared with available experimental data. ${ }^{6}$ Thus, there is confidence in the ability of the numerical method to accurately predict the trends at the higher freestream Mach numbers.

In this study, computational results are obtained for an isolated F/A-18 fuselage forebody at three freestream Mach numbers. No-blowing solutions are obtained to investigate the effects of Mach number on the baseline flowfields. The results obtained from the no-blowing solutions are compared with available experimental data. Two different active slot configurations are investigated at each freestream Mach number. Five different mass flow ratios (MFR) are used with each slot configuration (Table 1). MFR is defined as the ratio of the jet mass flow rate to a reference mass flow rate based on freestream density and velocity and the wing surface area. The results of the analysis provide an understanding of the effect of freestream Mach number on the efficiency of tangential slot blowing.
The next section briefly describes the numerical method, turbulence model, and grid system used in this investigation. The computational results are then presented and discussed. Conclusions are then drawn based on the analysis of the data.

\section{Numerical Method}

Since flow about a body at high angle of attack involves viscous effects and three-dimensional separated flow, the threedimensional Navier-Stokes equations must be solved to accurately resolve the relevant flow features. Solution of the three-dimensional thin-layer Navier-Stokes equations are obtained using the F3D code, reported by Steger et al. ${ }^{7}$ F3D is a two-factor, implicit, finite difference algorithm with an approximately factored, partially flux-split scheme. It uses upwind differencing in the streamwise direction and central differencing in the other two directions. It offers second-order accuracy in space and either first-or second-order accuracy in time. For the time-accurate computations reported in this study, first-order time accuracy is used. A complete description of the numerical method and the code may be found in Refs. 7 and 8 .

This code has been used extensively over the past several years to accurately predict the flowfield about the isolated F/A-18 forebody ${ }^{9}$ and full F/A-18 geometry ${ }^{10}$ at high angle of attack. The computed surface pressure coefficient obtained from solutions using the isolated forebody ${ }^{9}$ compared quite well with flight-test data, especially in the forebody barrel region. In the LEX region, discrepancies occurred due to the lack of geometry definition. Including the rear fuselage, wing, and empennage improved the comparison between the computed and experimental surface pressure coefficient. ${ }^{10}$ Previous computational results using the isolated forebody and wind-tunnel test conditions produced a good comparison of 
the surface pressure coefficients as well. ${ }^{6}$ These results indicate that the numerical method can accurately predict the effects of forebody tangential slot blowing on the flowfield and predict the amount of yawing moment generated by blowing.

Since the flowfields of interest are turbulent in nature, the Baldwin-Lomax algebraic turbulence model ${ }^{11}$ with modifications by Degani and Schiff ${ }^{12}$ is used. The Degani-Schiff modifications account for the separated flow and formation of vortices about bodies of revolution at high angle of attack. ${ }^{12}$ This turbulence model and modification were used in the previous computational studies of the F-18 isolated forebody and full aircraft with good results..$^{6,9} 10$ Although the BaldwinLomax turbulence model was not developed for use in the attached jet region, comparison of the computed and experimental surface pressure data indicates that the error introduced by the turbulence model is small and localized. ${ }^{6}$

The grid system used to model the isolated F-18 forebody in the present computations, shown schematically in Fig. 2, is similar to that used by Gee et al. ${ }^{6}$ The grid system consists of six grids and uses the overset grid method ${ }^{13}$ to facilitate boundary data transfer among the grids. The slot geometry is modeled in this grid system by the use of two grids in the nose of the forebody (Fig. 2). The physical slot geometry is patterned after the slot configuration used in the full-scale wind-tunnel experiments ${ }^{5}$ (Fig. 3). In the experimental setup, the slot was divided into six 8-in. segments individually connected to valves. In this way, the active slot length and location could be varied during the experiment. The jet length is varied in the computational results through the use of appropriate boundary conditions.

The jet is modeled computationally by using boundary conditions to introduce the jet exit conditions into the flowfield.

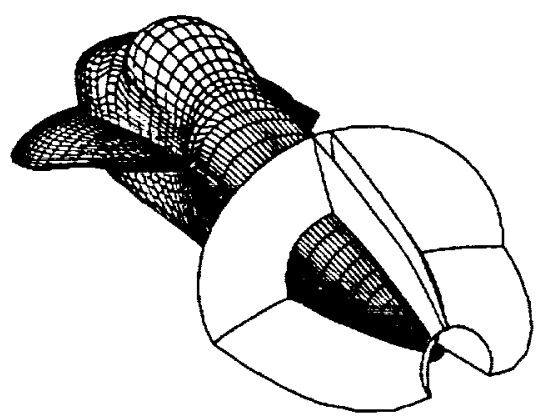

Fig. 2 Schematic of grid system used to model F/A-18 isolated forebody.

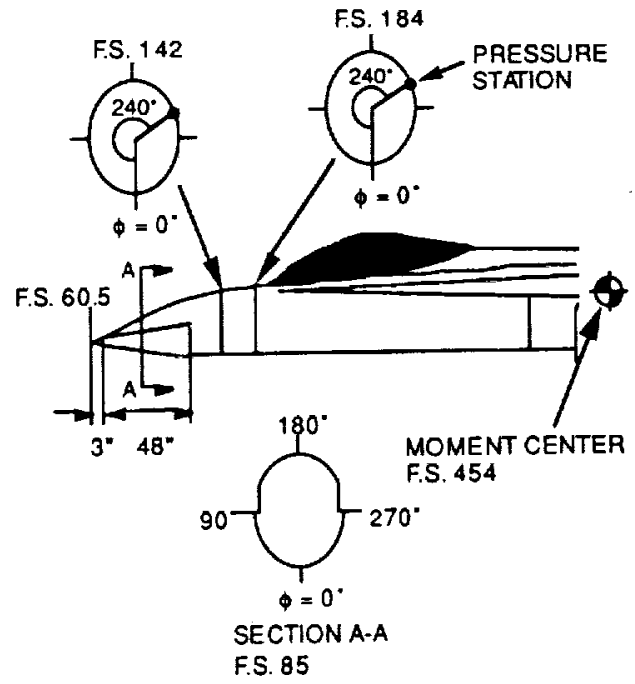

Fig. 3 Schematic of the slot configuration modeled in grid system.
If the jet exit Mach number is less than sonic, the jet total pressure and total temperature are input into the flow solver. The exit pressure is obtained by extrapolating the pressure from the local external flow pressure at the jet exit and the jet exit Mach number is obtained using the isentropic relations. For sonic flow, the jet is assumed to choke at the exit and the jet exit pressure is obtained from isentropic relations using the jet total pressure and temperature inputs. In either case, in order to obtain the desired MFR value, the total pressure of the jet is increased, thereby increasing the jet density, until the desired jet mass flow rate is obtained. In addition, a no-slip boundary condition is applied at the forebody surface, freestream conditions are maintained at all inflow boundaries, and a zero-gradient extrapolation in the axial direction is used at the exit boundary.

\section{Results and Discussion}

One objective of the computational investigation is to determine the effect of freestream Mach number on the efficiency of tangential slot blowing. Therefore, computed noblowing and blowing solutions are obtained for flow about an isolated F-18 forebody at $\alpha=30.3 \mathrm{deg}$ at three different freestream Mach numbers, $M_{x}=0.243,0.400$, and 0.700 . The corresponding Reynolds numbers, based on the F/A-18 wing mean aerodynamic chord, are $R e_{\bar{c}}=11.0 \times 10^{6}, 18.0$ $\times 10^{6}$, and $31.4 \times 10^{6}$, respectively.

\section{No-Blowing Solutions}

No-blowing solutions are obtained at each freestream Mach number and serve as baseline solutions from which the blowing solutions are computed. Analysis of the no-blowing solutions also serves as a check to insure that the numerical method is accurately predicting the flowfields and the relevant trends. Although details of the flowfield are similar to results presented previously, ${ }^{6}$ the main features are briefly discussed for comparison with the blowing results.

\section{Flowfield Characteristics}

Figure 4 shows the surface flow pattern and off-surface instantaneous streamlines obtained from the solution computed at $M_{x}=0.700$. The flowfield is similar to that reported in previous work with the isolated F/A-18 forebody at a lower freestream Mach number. ${ }^{6}$ There are a primary and secondary separation line on each side of the forebody barrel. Flow that separates from the forebody rolls up to form vortices above the forebody (Fig. 4b). Each wing leading-edge extension (LEX) has a sharp leading edge and a primary crossflow separation line lies along this edge. A secondary separation line is also evident on the upper surface of each LEX (Fig. 4a). At this angle of attack, the no-blowing flowfield is symmetric. a)

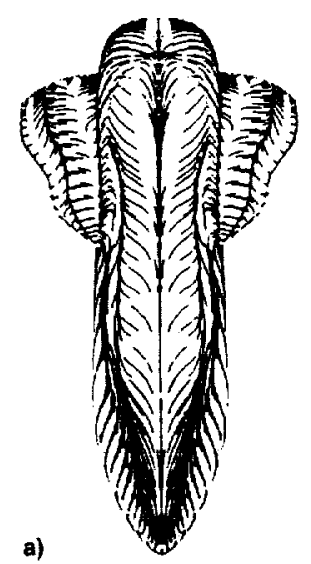

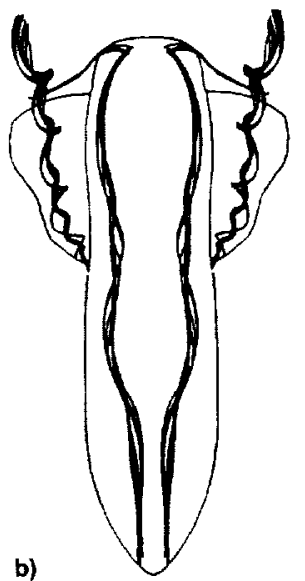

b)
Fig. 4 Flowfield characteristics, $M_{x}=0.700, \alpha=30.3 \mathrm{deg}, R e_{\bar{c}}=$ $31.4 \times 10^{6}$ : a) surface flow pattern and $b$ ) off-surface instantaneous streamlines. 


\section{Surface Pressure Coefficient Comparison}

Figure 5 shows a comparison of the computational and experimental ${ }^{14}$ spanwise surface pressure distributions for each Mach number case at three axial locations on the LEX. The computed results presented here are obtained with a corrected set of boundary conditions, and are different from the results presented in the meeting paper. ${ }^{15}$ Experimental data show a reduction in the suction peaks with increasing freestream Mach number. ${ }^{14}$ This trend is also evident in the computational results. The accuracy of the computed results increases as the freestream Mach number increases. The symmetric nature of the flowfield is evident in both the computational and experimental data. At the first two LEX stations, the computed results at $M_{\mathrm{x}}=0.243$ underpredict the suction peaks slightly. This is due to the difference between the computed and experimental freestream Mach number. The suction peaks are accurately predicted in the other two Mach number solutions, where the computed and experimental freestream Mach numbers match.
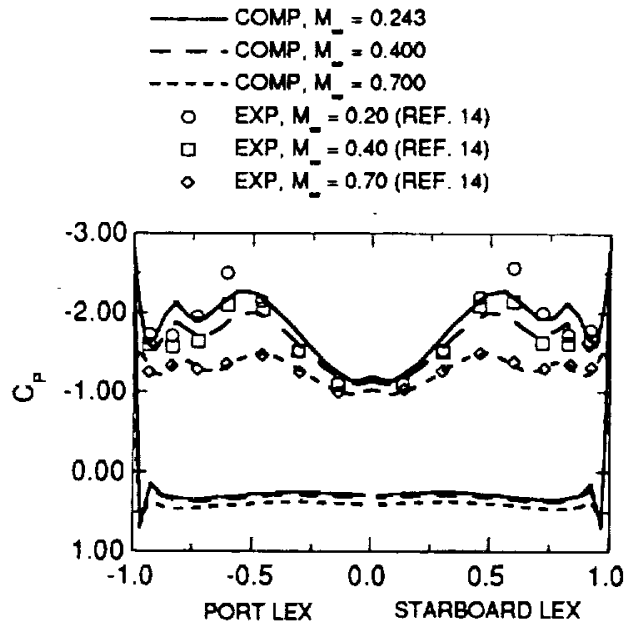

a)

$y / B$

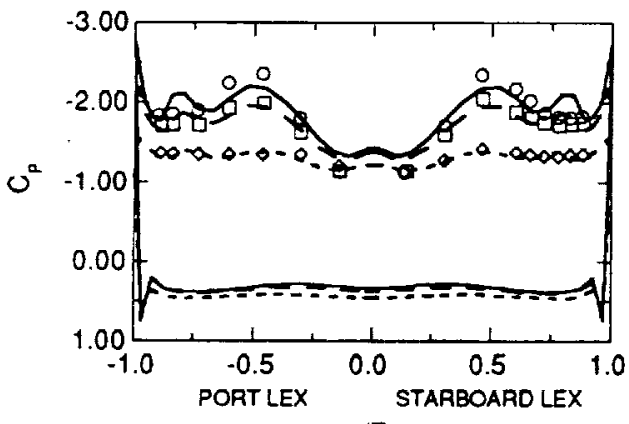

b)

y/B

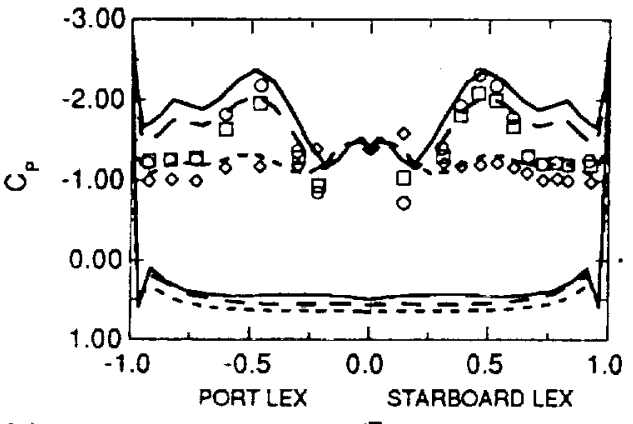

c) y/B

Fig. 5 Comparison of computed surface pressure coefficient; $\alpha=$ 30.3 deg: a) F.S. 253, b) F.S. 296, and c) F.S. 357.
At the aftmost LEX station, the computed results slightly overpredict the suction peak at all three Mach numbers. The isolated forebody computations cannot resolve the LEX vortex burst due to the lack of geometry definition. Addition of the wing and tail geometry produced a better comparison with flight test data." By including the wing and tail, LEX vortex burst is resolved. This affects the surface pressure, especially at the last pressure station, F.S. 357, since the burst occurs in this region. The overall good agreement in the trends and surface pressure comparison shown in the no-blowing solutions provide confidence that the analogous trends seen in the computed blowing solutions will also be valid.

\section{Blowing Solutions}

Solutions with blowing are obtained at each freestream Mach number using two active slot configurations. One configuration consists of a 16-in. active slot beginning 11 in. aft of the nose (hereafter referred to as the 16-11 in. slot). The other slot configuration has a 24-in. slot beginning $3 \mathrm{in.} \mathrm{aft} \mathrm{of} \mathrm{the}$ nose (24-3 in. slot). Blowing occurs only on the port side (pilot's view) of the forebody. For each slot configuration and freestream Mach number, solutions are obtained at five MFRs ranging from $0.03 \times 10^{-3}$ to $0.24 \times 10^{3}$ (Table 1). At $M_{x}$ $=0.243$, additional cases are computed for MFR $=0.015 \times$ $10^{-3}$. The results permit evaluation of the effect of varying Mach number, at a fixed MFR, on the efficiency of tangential slot blowing, as well as the effect of varying MFR at a fixed Mach number.

\section{Yawing Moment Comparison}

The yawing moment $C_{n}$, obtained from blowing, is plotted against MFR for both slot configurations in Fig. 6 . The moment center used to compute $C_{n}$ is located at the c.g. point of the aircraft, F.S. 454 (Fig. 3). As was seen previously in subscale $^{4}$ and full-scale ${ }^{5}$ wind-tunnel tests, the MFR is a good parameter for correlating the forces produced by blowing at differing flow conditions.

The computed results show that both slot configurations are capable of generating yawing moment, even at transonic
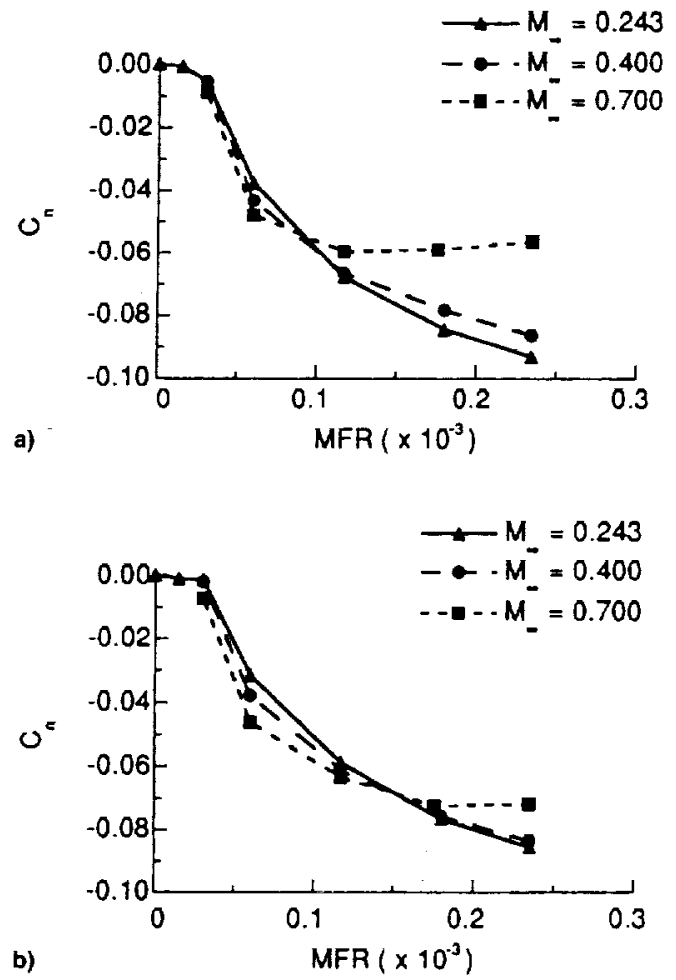

Fig. 6 Computed yawing moment plotted against MFR for isolated forebody with blowing; $\alpha=30.3 \mathrm{deg}$ : a) $16-11$ and b) $24-3$ in. slots. 
maneuvering Mach numbers. For both slot configurations at $M_{\text {x }}=0.243$ and 0.400 , the yawing moment increases with increasing MFR. For the case with the $16-11$ in. slot at $M_{x}$ $=0.700$, the yawing moment first increases, then levels off and decreases slightly as the MFR increases. A similar, but less pronounced, leveling off of $C_{n}$ also occurs for the 24-3 in. slot at $M_{x}=0.700$. However, useful yawing moments are obtained at moderate jet mass flow rates at all freestream Mach numbers (Table 1). Further analysis of the computed flowfields yields information about the flow physics associated with the behavior of the curves shown in Fig. 6 .

At the lowest blowing rate analyzed, MFR $=0.015 \times 10^{3}$, almost no yawing moment is obtained for either slot configuration. This is consistent with the subscale results obtained by Kramer et al. ${ }^{4}$ At this angle of attack, no force reversal was observed in either the experimental or computational data.

The computed surface flow pattern and off-surface instantaneous streamlines, obtained from the 16-11 in. slot, $M_{x}=$ 0.243 , MFR $=0.015 \times 10^{-3}$ solution (Fig. 7 ), show the jet separating along with the blowing-side primary forebody vortex. There is no change in the position of the blowing-side primary separation line on the forebody barrel (Fig. 7a). The off-surface instantaneous streamlines (Fig. 7b) show the jet to have almost no effect on the position of either the blowingside or non-blowing-side forebody vortex. The early separation reduces the low-pressure region caused by the attached jet and reduces the interaction of the jet with the non-blowingside forebody vortex. Both of these effects serve to reduce the amount of side force and yawing moment generated.

At MFR $=0.03 \times 10^{3}$, blowing from the 16-11 in. slot generates slightly higher amounts of $C_{n}$ than blowing from the 24-3 in. slot. The smaller area of the $16-11$ in. slot requires a higher jet exit Mach number to obtain a given jet mass flow rate. The higher jet exit velocity increases the suction pressure generated by the attached portion of the jet. This serves to increase the yawing moment generated by blowing.

At MFR $=0.06 \times 10^{-3}$, the yawing moment increases slightly with increasing freestream Mach number. This is most evident in the 24-3 in. slot configuration results. Again, this is due to the differences in the jet exit Mach numbers (Table 1). As the freestream Mach number increases, the jet mass flow rate must increase to maintain a given MFR value. An increase in jet mass flow rate causes a corresponding increase in the jet exit Mach number until choked conditions are reached at the slot exit.

Once the jet is choked, the effectiveness of blowing depends upon the jet exit pressure. The ratio of $P_{e}$ to $P_{u}$ is presented in Table 1. For moderate values of this ratio $P_{\mathrm{c}} / P_{a}<1.5, C_{n}$
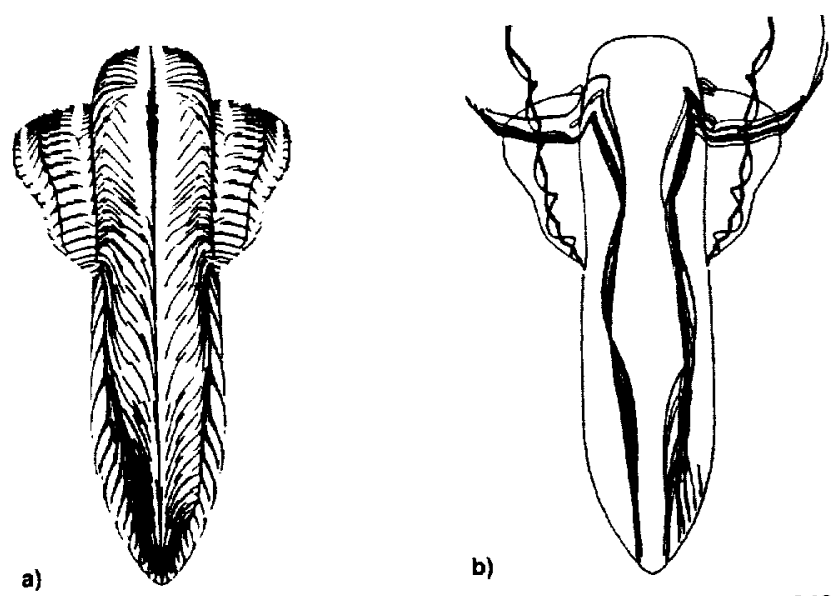

Fig. 7 Flowfleld characteristics at low blowing rates. $M_{x}=0.243$, Fig. 7 Flowfield characteristics at low $0^{-6}, \mathrm{MFR}=0.015 \times 10^{-3}, 16-11 \mathrm{in}$. $\alpha=30.3 \mathrm{deg}, R e_{i}=31.4 \times 10^{-6}$, MFR $=0.015 \times 10^{-3}, 16-11 \mathrm{in}$. lines. increases with MFR and does not depend on the freestream Mach number. This can be seen in 24-3 in. slot results for $0.12 \times 10^{-3}<$ MFR $<0.24 \times 10^{3}$. However, for $P_{e} / P_{a}>$ 1.5 , the blowing effectiveness levels off. This is most evident in the $16-11$ in. slot, $M_{x}=0.700$ case. As the blowing rate, and thus the jet exit pressure, increases, the yawing moment levels off and slightly decreases for this case. This is due to the phenomenon of overblowing.

\section{Effects of Overblowing}

Overblowing has been observed experimentally ${ }^{4}$ as a dropoff of yawing moment at high blowing rates. The effect of overblowing on the computed flowfield is observed by plotting the velocity vectors in a crossflow plane at F.S. 85 that passes through the jet region (Fig. 8). The leveling off and reduction of the yawing moment observed in the overblowing region is due mainly to the early separation of the jet. Overblowing occurs when the jet flow is sonic and underexpanded $\left(P_{p} / P_{a}\right.$ $>1.0$ ) at the slot exit. For $P_{e} / P_{u}>1.5$, the jet rapidly expands after leaving the slot, deflecting the flow away from the fuselage surface, causing earlier crossflow separation. This action negates the Coanda effect, which causes delay of the crossflow separation. At the lower blowing rate (Fig. 8a), the jet remains attached to the surface. As the jet negotiates the curvature of the surface, the surface pressure drops, generating a low-pressure region, contributing to the side force and yawing moment generated. However, in a case with overblowing, the jet does not remain attached to the surface (Fig. 8 b). Rather, it separates and rides on top of a layer of fluid that is moving in the opposite direction. The separation of the jet reduces the suction generated by the jet, thereby reducing the side force and yawing moment. Side force and yawing moment are still generated due to the manipulation of the forebody vortices by the jet.

The behavior of the overblown jet is observed graphically using instantaneous streamlines to illustrate the vortices formed on the nose and the jet (Fig. 9). For the attached jet flow
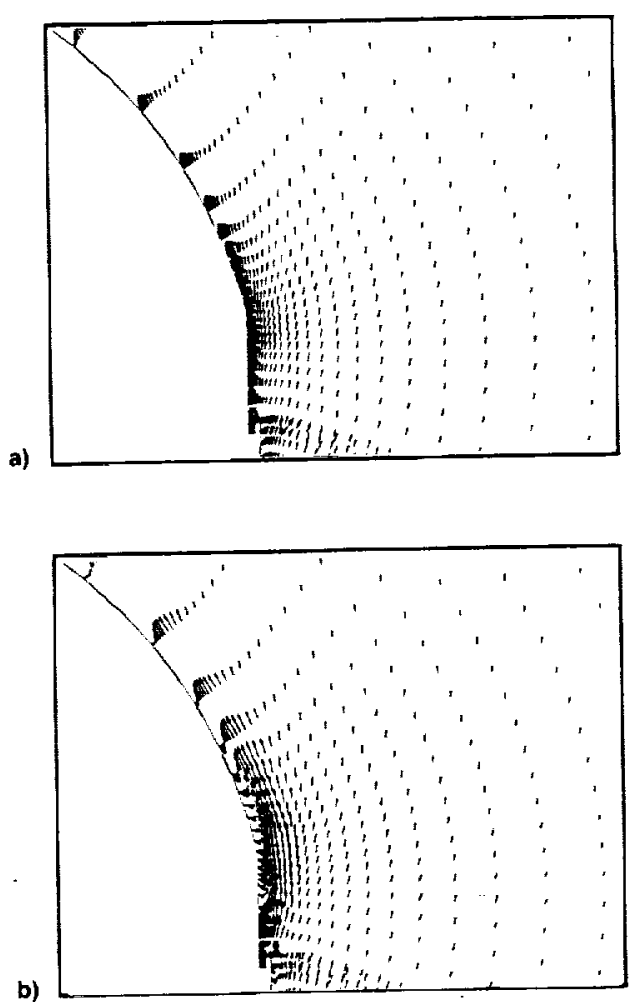

Fig. 8 Effect of overblowing on flow in vicinity of the slot; computed velocity vectors in the crossflow plane at F.S. 85, $M_{x}=0.700, \alpha=$ $30.3 \mathrm{deg}, R e_{c}=31.4 \times 10^{6}, 16-11$ in. slot. $\mathrm{MFR}=$ a) $0.06 \times 10^{-3}$, $P_{e} / P_{a}=1.50$ and b) $0.24 \times 10^{-3}, P_{e} / P_{a}=5.86$. 


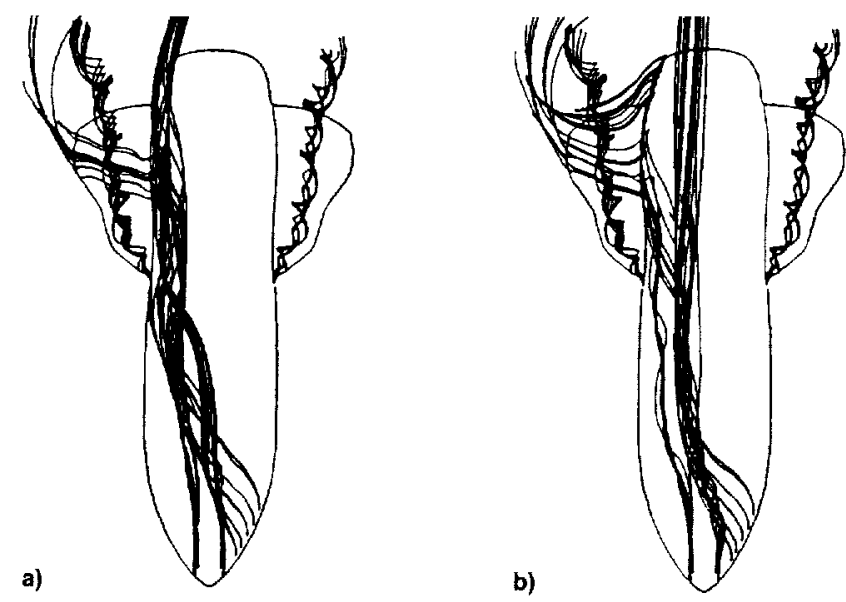

Fig. 9 Off-surface instantaneous streamlines with blowing. $M_{*}=$ $0.700, \alpha=30.3 \mathrm{deg}, R_{e_{r}}=31.4 \times 10^{6}, 16-11$ in. slot. MFR $=$ a) $0.06 \times 10^{-3}, P_{f} / P_{a}=1.50$ and b) $0.24 \times 10^{-3}, P_{e} / P_{a}=5.86$.

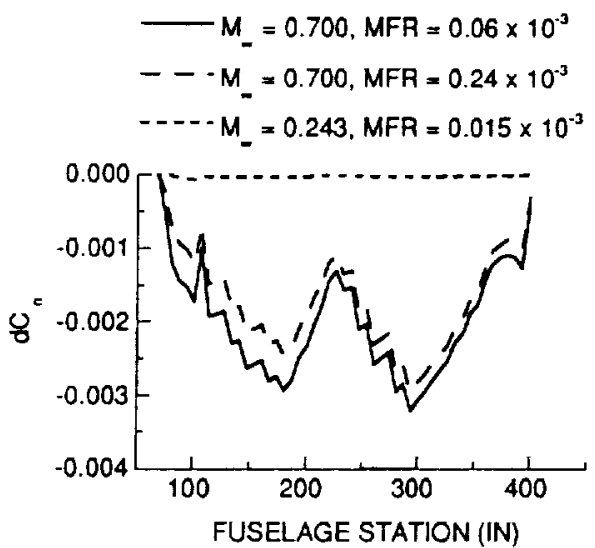

Fig. 10 Computed local yawing moment distribution with blowing, 16-11 in. slot.

(Fig. 9a), blowing causes the nose vortex on the blowing side to merge with the nose vortex on the nonblowing side. The jet flow also becomes entwined in this merged nose vortex. In the overblown case (Fig. 9b), the two nose vortices do not merge, although there is still a slight interaction between the jet flow and the non-blowing-side nose vortex. This is in contrast to the very low-blowing case (Fig. 7b), where no interaction between the jet and non-blowing-side forebody vortex is observed.

The behavior of the jet also has an effect on the contribution of the forebody barrel and LEX region to the yawing moment. This effect can be seen in Fig. 10, which presents the local yawing moment distribution along the forcbody. Previous computational studies $s^{6,16}$ indicated that there is a contribution to the side force and yawing moment from the forebody barrel aft of the slot and the LEX region. At the lowest blowing rate shown, there is almost no yawing moment evident along the entire forebody. This is due in part to the early separation of the jet. Without this flow interacting with the non-blowingside LEX vortex, changes in the surface pressure in the LEX region are reduced. Overblowing reduces the amount of yawing moment obtained in the blowing region as well as over the remainder of the forebody. Again, this is due to the early separation of the jet and the limited interaction between the jet and the non-blowing-side nose and LEX vortices.

The phenomenon of overblowing can be avoided by limiting the jet exit pressure to 1.5 times the local static pressure in the slot region. This can be accomplished at high jet mass flow rates by increasing the area of the slot. At the high blowing rates, the larger area of the $24-3$ in. slot is beneficial
(Fig. 6b), since a lower jet total pressure is required to obtain a given MFR (Table 1). Overblowing starts at MFR $=0.12$ $\times 10^{3}$ for the $16-11$ in. slot; for the $24-3$ in. slot, the onset of overblowing does not occur until MFR $=0.24 \times 10^{3}$. For both slot configurations, the computed results indicate that blowing can generate useful amounts of yawing moment at moderate blowing rates, even at transonic Mach numbers.

\section{Force Onset Time Lag}

Time-accurate solutions are obtained using the isolated $F$ / A-18 forebody, the 16-11 in. slot configuration, and MFR = $0.06 \times 10^{3}$ to determine the force onset time lag associated with forebody tangential slot blowing. The forebody $C_{n}$ are plotted against $\bar{t}$ in Fig. 11 . Blowing is activated at $\bar{t}=0.0$ in all cases. The time lag associated with charging up the plenum chamber and associated plumbing is not modeled. The yawing moment coefficient time histories (Fig. 11) show that it requires about one nondimensional time unit for the yawing moment to reach a maximum steady value. This time lag is consistent with data obtained in subscale ${ }^{4}$ and full-scale wind-tunnel tests. In all cases, the flowfield has reached its steady-state value in the time required for the freestream flow to traverse approximately three mean aerodynamic chord lengths, which corresponds to the length of the isolated forebody used in the present computations.

The time lag is also studied by examining the surface-pressure coefficient at two axial locations on the forebody barrel (Fig. 12). The two points are located on the forebody barrel

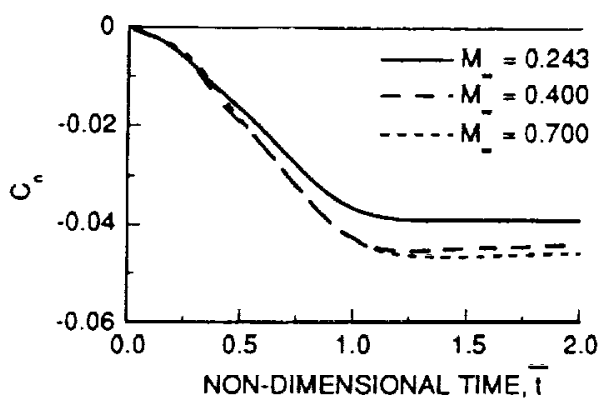

Fig. 11 Time history of forebody yawing moment. $M_{\star}=0.243, \alpha$ $=30.3 \mathrm{deg}, R e_{\bar{r}}=11.0 \times 10^{n}, \mathrm{MFR}=0.06 \times 10^{-3}, 16-11 \mathrm{in}$. slot.
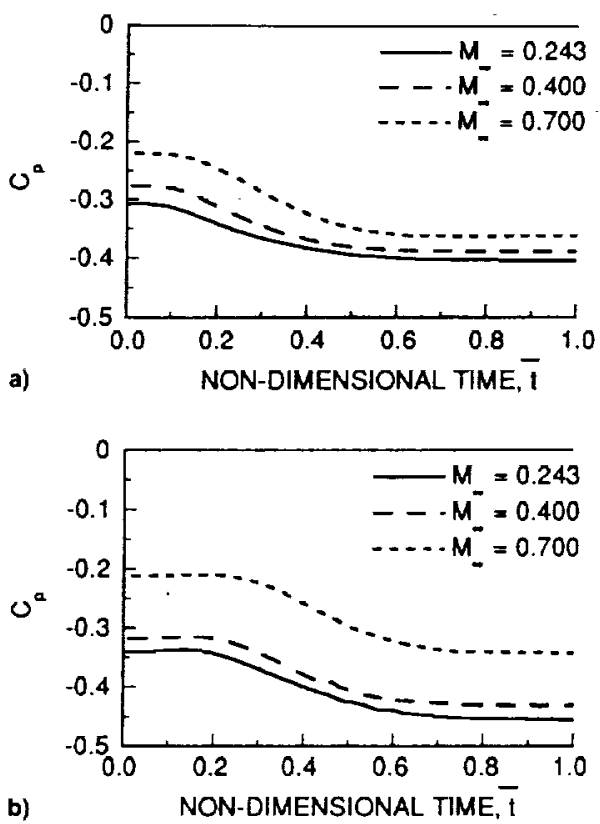

Fig. 12 Time history of surface pressure coefficient. $M_{x}=0.243$, $\alpha=30.3 \mathrm{deg}, R e_{\mathrm{s}}=11.0 \times 10^{\circ}, \mathrm{MFR}=0.06 \times 10^{-3}, 16-11 \mathrm{in}$. slot: a) F.S. 142, $\phi=240 \mathrm{deg}$ and b) F.S. $184, \phi=240 \mathrm{deg}$. 
on the blowing side of the body, as shown in Fig. 3. At F.S. 142 , for $M_{x}=0.243$ (Fig. 12a), the computed data shows a delay of about 0.1 nondimensional time units, or 0.01 -s real time, followed by a ramp down of the surface pressure over a period of 0.5 nondimensional time units $(0.065$-s real time). This behavior is also seen in the experimental data. ${ }^{5}$ As the freestream Mach number increases, the response time decreases. At F.S. 184 (Fig. 12b), the response times for the $M_{*}=0.243$ case increase to 0.2 nondimensional time units $(0.025 \mathrm{~s})$ and 0.5 nondimensional time units $(0.065 \mathrm{~s})$ for the delay and ramp down, respectively. Again, the response time decreases with increasing Mach number. This data indicates that the time lags associated with development of yawing moments using pneumatic slot blowing for forebody flow control are not large enough to be detrimental to the usefulness of the system.

\section{Conclusions}

A computational analysis of the effect of freestream Mach number on the effectiveness of forebody tangential slot blowing was presented. The flow about an isolated F-18 forebody was computed using a thin-layer Navier-Stokes flow solver. Solutions were obtained at three different freestream Mach numbers. At each Mach number, two slot geometries and five different MFRs were used. Additional solutions were obtained at the lowest freestream Mach number using an even lower MFR. Time-accurate solutions were obtained to determine the force onset time lag due to blowing.

The computational results indicated that forebody tangential slot blowing remained effective, even at transonic Mach numbers. At the very low MFRs, blowing had no effect on the flowfield. The jet separated along the primary separation line seen in the no-blowing solution and did not change the position of the forebody vortices. As the MFR increased, the yawing moment generated increased. At a given MFR, the yawing moment increased with increasing freestream Mach number. This was due to the increase in the jet exit velocity. As the jet exit velocity became sonic, this effect diminished. Further increases in the MFR lead to overblowing. This was especially evident at the highest freestream Mach number and highest MFR value analyzed. Overblowing was caused by the jet being underexpanded as it left the slot. The rapid expansion of the jet caused the jet to separate from the surface. This early separation reduced the effectiveness of the pneumatic system. Unlike the low blowing rate cases, the overblown jet still had an effect on the position of the vortices and generated a significant yawing moment. Overblowing was avoided by limiting the jet exit pressure ratio. For high jet mass flow rates, this was achieved by increasing the slot area. The results showed that tangential slot blowing remained effective at transonic Mach numbers.

Time-accurate solutions were obtained using one of the slot configurations, one MFR, and all three freestream Mach numbers. The yawing moment time history and the surface pressure coefficient time history at two points on the forebody barrel were recorded for each case. The yawing moment history indicated that a steady-state value was reached in the time required for a particle in the flowfield to travel approx- imately three mean aerodynamic chord lengths. The surface pressure coefficient indicated a small delay followed by a ramp down in pressure as the jet was convected downstream. These time lags were of the same order as those measured in fullscale and subscale wind-tunnel tests. The results indicated that the time lags did not present an obstacle to implementation of forebody tangential slot blowing on an aircraft.

\section{References}

'Ng, T. T., and Malcolm, G. N., "Aerodynamic Control Using Forebody Blowing and Suction," AIAA Paper 91-0619, Jan. 1991.

"Gee, K., Tavella, D., and Schiff, L. B., "Computational Investigation of a Pneumatic Forebody Flow Control Concept," Journal of Aircrafi, Vol. 30, No. 3, 1993, pp. 326-333.

${ }^{3} \mathrm{Ng}$, T. T., Suarez, C. J., and Malcolm, G. N., "Forebody Vortex Control Using Slot Blowing," Proceedings of the 9th Applied Aerodynamics Conference, 1991, pp. 412-422 (AIAA Paper 91-3254).

${ }^{4}$ Kramer, B., Suarez, C., and Malcolm, G., "Forebody Vortex Control with Jet and Slot Blowing on an F/A-18," AIAA Paper 933449. Aug. 1993.

'Lanser, W. R., Meyn, L. A., and James, K. D., "Forebody Flow Control on a Full-Scale F/A-18 Aircraft," AIAA Paper 92-2674, June 1992.

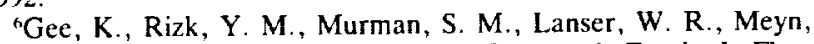
L. A., and Schiff, L. B., "Analysis of a Pneumatic Forebody Flow Control Concept About a Full Aircraft Geometry," AIAA Paper $92-$ 2678, June 1992.

${ }^{7}$ Steger, J. L., Ying, S. X., and Schiff, L. B., "A Partially FluxSplit Algorithm for Numerical Simulation of Compressible Inviscid and Viscous Flow," Proceedings of a Workshop on Computational Fluid Dynamics, Univ. of California, Davis, CA, 1986.

${ }^{\mathrm{x}}$ Ying, S. X., Schiff, L. B., and Steger, J. L., "A Numerical Study of Three-Dimensional Separated Flow Past a Hemisphere Cylinder," AIAA Paper 87-1207, June 1987

"Cummings, R. M., Rizk, Y. M., Schiff, L. B., and Chaderjian, N. M. "Navier-Stokes Predictions for the F- 18 Wing and Fuselage at Large Incidence," Journal of Aircraft, Vol. 29, No. 4, 1992, pp. $565-574$.

"Rizk, Y. M., and Gee, K., "Unsteady Simulation of Viscous Flowfield Around F-18 Aircraft at Large Incidence," Journal of Aircraft, Vol. 29, No. 6, 1992, pp. 986-992.

"Baldwin, B., and Lomax, H., "Thin-Layer Approximation and Algebraic Model for Separated Turbulent Flows," AIAA Paper 780257, Jan. 1978.

"Degani, D., and Schiff, L. B., "Computation of Turbulent Supersonic Flows About Pointed Bodies Having Crossflow Separation," Journal of Computational Physics, Vol. 66, No. 1, 1986, pp. 183196.

${ }^{13}$ Benek, J. A., Steger, J. L., Dougherty, F. C., and Buning, P. G. "Chimera: A Grid Embedding Technique," Arnold Air Force Station, Arnold Engineering Development Center, AEDC-TR-8564, 1986.

"Erickson, G. E., "Wind Tunnel Investigation of Vortex Flows on F/A-18 Configuration at Subsonic Through Transonic Speeds," NASA TP 3111, Dec. 1991

"Gee, K., Rizk, Y. M., and Schiff, L. B., "Analysis of Tangential Slot Blowing on F/A-18 Isolated Forebody," AIAA Paper 94-1831, June 1994.

${ }^{16 G e e}$, K., Rizk, Y. M., and Schiff, L. B., "Effect of Forebody Tangential Slot Blowing on an Aircraft Geometry," Journal of Aircraft, Vol. 31, No. 4, 1994, pp. 922-928. 